\title{
Characterization of novel microneme adhesive repeats (MAR) in Eimeria tenella
}

\author{
Virginia Marugan-Hernandez, Rebekah Fiddy, Jazmine Nurse-Francis, Oliver Smith, Laura Pritchard \\ and Fiona M. Tomley
}

\begin{abstract}
Background: The phylum Apicomplexa comprises a wide variety of parasites of significant medical and economic relevance. These parasites have extremely different host and tissue tropisms; for example Toxoplasma gondii can invade virtually any nucleated cell and infect almost all warm-blooded vertebrates, whereas Eimeria tenella infects only chickens and is restricted in its growth to epithelial cells of the caecum. Proteins released from the microneme secretory organelles (MICs) are critical for apicomplexan invasion of host cells and allow parasites to bind a diverse range of host cell oligosaccharide epitopes. MICs bear modular arrangements of sequences with adhesive proteins and interestingly the sialic-acid binding MAR (microneme adhesive repeat) domain containing proteins (MCPs) are suggested to make significant contributions to the different host and tissue tropisms of T. gondii and E. tenella.

Results: In this study, we evaluated the binding capacity of Type I MAR domains from novel E. tenella MCPs. Variants of the previously described HxT motif were analysed showing that HxT and VxT variants bind, whereas HxS and YXE variants did not. One of these MCP containing a single MAR (EtMCP2) showed an apical localization when expressed as a fusion with the fluorescent reporter mCherry in transgenic populations and a similar pattern of transcripts per zoite during endogenous development in vitro as the well-characterised microneme protein EtMIC2.

Conclusions: Variation in the binding properties of the MAR of different EtMCPs was confirmed and their ability to bind a wider range of sialic acids and terminal linkages should be studied. In addition, transgenesis technology has been used for first time in Eimeria parasites as a rapid tool for the study of endogenous protein localization by fusion with a fluorescent reporter.
\end{abstract}

Keywords: Eimeria tenella, Microneme proteins, Binding domains, Parasite-host interaction

\section{Background}

Seven species of the genus Eimeria (Apicomplexa, Coccidia) cause chicken coccidiosis, a disease with a huge economic impact in the poultry industry. Disease pathology is characterised by diarrhoea, malabsorption and for some species haemorrhage, and has a severe impact on animal welfare, efficiency of feed conversion and weight gain. Eimeria parasites disseminate readily through flocks via the oral-faecal route and are highly prevalent throughout the world [1]. Coccidiosis control relies mainly on in-feed anticoccidial drugs; however, drug resistance is ubiquitous and there are increasing concerns from consumers regarding risks of drug residues entering the food chain. Immune-

\footnotetext{
*Correspondence: vhernandez@rvc.ac.uk

Department of Pathobiology and Population Sciences, The Royal Veterinary College, University of London, Hawkshead Lane, North Mymms, Hatfield, Hertfordshire AL9 7TA, UK
}

prophylaxis using live-attenuated vaccines is also effective for coccidiosis control and is widely used in breeding and laying flocks. However, because the parasites do not replicate outside of their host, vaccine production requires large numbers of chickens to amplify lines of vaccinal parasites; this places a practical limitation on production and means that vaccines are costly compared to anticoccidial drugs [2].

Understanding the biology of Eimeria parasites in order to elucidate the functional significance and immunogenicity of parasite sub-cellular structures aids discovery of antigens for new types of vaccines. A substantial amount of data from high-throughput technologies is now available [3-5]. However, there is a clear imbalance between these new data and our capacity to validate the role of specific proteins in key biological processes. Conventional methods for protein characterisation are time-consuming, expensive and not 
always conclusive. Complementary technologies based on reverse genetics are available for some apicomplexan parasites, including the coccidian Toxoplasma gondii [6], and are important tools to study gene functionality. For example, gene knock-in and knock-out has been used to investigate parasite invasion and protein trafficking $[7,8]$ and to elucidate the functional role of several vaccine antigens including TgAMA1 and TgROP18 $[9,10]$. Reverse genetics tools for Eimeria spp. are less developed, however, efficient random integration of transgenes is well established [11, 12] for expressing and targeting of reporter molecules and heterologous antigens [13-17].

Proteins secreted from apicomplexan microneme organelles (MICs) play important roles in parasite adhesion and invasion of host cells [18]. Some MIC proteins are specific for coccidial parasites and mediate parasite recognition of host cell molecules. These include a family of MICs that contain copies of a protein domain termed microneme adhesive repeat (MAR) that binds sialic acid $[19,20]$. Binding to the sialyl ligand is co-ordinated through hydrogen bonding and pi-stacking to histidine and threonine residues in a HxT motif that is conserved across many, but not all, MAR [19, 21]. All MAR domains adopt the same structural fold, but they are subclassified into type I and type II MAR on the basis of their primary sequence, with type I having an extended a1helix/loop region and (some) type II having an additional C-terminal $\beta$-finger region [21]. These subtle structural differences, in addition to specific sequence divergence within individual
MAR, confer differential binding properties. In particular, bulky side chains in the extended a1helix/loop region of Type I MAR (specifically a LxxY motif in MAR from the E. tenella protein EtMIC3) make increased contacts to the sialyl saccharide leading to a higher level of specificity in binding, and precluding interaction with $\mathrm{N}$-glycolyl sialic acids [22].

Toxoplasma gondii and Neospora caninum possess proteins with both Type I and Type II MAR. However, to date all MAR domains identified in Eimeria species are exclusively Type I, suggesting that a lack of Type II MAR may contribute to the exquisite host and site specificity displayed by these parasites [21, 22]. For Eimeria tenella, EtMIC3 containing seven Type I MAR binds a restricted range of sialyl glycans with a strong preference for a2,3-linkages that are predominant in the chicken gut [22, 23]. Four additional Type I MAR containing proteins (EtMCP2 with 1 MAR; EtMCP3 with 3 MAR; EtMCP4 with 4 MAR, EtMCP5 with 2 MAR) are present in the E. tenella genome [21] (Table 1) but their subcellular location, stage-expression and function are unknown. Whilst five of the seven Type I MAR of EtMIC3 have the conserved sialic acid binding motif HxT, this is present in only two of the ten Type I MAR of the EtMCPs, with the remainder having partial or no conservation of the motif.

In this paper, we examine the ability of EtMCP Type I MARs with variant $\mathrm{HxT}$ motifs to bind in vitro to fetuin-agarose or to cultured cells. To explore the subcellular location of an MCP with a variant, non-binding

Table 1 Summary of E. tenella MCPs and their binding properties

\begin{tabular}{|c|c|c|c|c|}
\hline Repeat & Accession number & Motif & Recombinant expression & Fetuin / MDBK binding \\
\hline EtMCP2.1 & ETH_00020995 & $H-L-S$ & recMCP2.1 & No \\
\hline EtMCP3.1 & ETH_00030520 & $H-W-S$ & - & - \\
\hline EtMCP3.2 & & $V-W-T$ & - & - \\
\hline EtMCP3.3 & & $H-W-T$ & recMCP3.3 & Yes \\
\hline EtMCP4.1 & ETH_00030525 & $V-W-S$ & - & - \\
\hline EtMCP4.2 & & $H-W-S$ & recMCP4.2 & No \\
\hline EtMCP4.3 & & $V-W-T$ & recMCP4.3 & Yes \\
\hline EtMCP4.4 & & $H-W-T$ & - & - \\
\hline EtMCP5.1 & ETH_00003280 & $X-X-X^{a}$ & - & - \\
\hline EtMCP5.2 & & $X-X-X^{a}$ & recMCP5.2 & No \\
\hline EtMIC3.1 & ETH_00021010 & $Y-L-T$ & - & - \\
\hline EtMIC3.2 & & $H-L-T$ & - & - \\
\hline EtMIC3.3 & & $H-L-T$ & recMIC3.3 & Yes \\
\hline EtMIC3.4 & & $H-L-T$ & - & - \\
\hline EtMIC3.5 & & $H-L-T$ & - & - \\
\hline EtMIC3.6 & & $H-L-T$ & - & - \\
\hline EtMIC3.7 & & $X-X-X^{a}$ & - & - \\
\hline
\end{tabular}

${ }^{\mathrm{a}} \mathrm{X}-\mathrm{X}-\mathrm{X}=$ motif not identifiable 
motif (EtMCP2), we generated a transgenic population of E. tenella parasites that overexpresses EtMCP2 as a mCherry fusion. We show that this fusion localises to the apical tip of the sporozoites, in a pattern similar to that of a transgenic population overexpressing mCherry fused to the known microneme protein EtMIC2. Furthermore, using wild type parasites we show by quantitative PCR (qPCR) and reverse-transcription qPCR (RT-qPCR) that EtMCP2 transcript/genome ratio during sporozoite invasion and intracellular schizogony is similar to that of the known microneme proteins, EtMIC2 and EtMIC5. Thus, despite an inability to bind sialyl moieties, the Type I MAR protein, EtMCP2, appears to be expressed within the microneme organelles of E. tenella.

\section{Methods}

\section{Parasites and birds}

Eimeria tenella Wisconsin strain [24] was propagated in vivo in 3 week old Lohmann White chickens obtained from APHA Weybridge, where they were produced and reared under specific pathogen free conditions. Oocyst harvesting and cleaning [25], and sporozoite excystation and purification [26] were carried out as previously described. For in vitro cell culture assays, purified sporozoites (wild type or transgenic) were added to confluent MDBK cell monolayers in 24-well plates $\left(0.3 \times 10^{6}\right.$ cells/ well) at a multiplicity of infection of $1: 1$ and incubated at $41{ }^{\circ} \mathrm{C} / 5 \% \mathrm{CO}_{2}$ for $4,24,48$ and $72 \mathrm{~h}$.

\section{Expression and purification of recombinant MAR regions in $E$. coli}

Complementary DNA (cDNA) sequences corresponding to protein repeats containing MAR with varying $\mathrm{HxT}$ motifs (Table 1) were amplified from parasite cDNA using the primers listed in Additional file 1: Table S1 and cloned between $\mathrm{NcoI}$ and NotI restriction sites into the plasmid pET32b (+) (Novagen, Hertfordshire, UK). The recombinant proteins were expressed as soluble polyhistidine (His6) and thioredoxin fusions in BL21 (DE3) pLysS E. coli (Stratagene, California, USA) and were purified and quantified as described previously [17].

\section{Fetuin-agarose affinity binding assay}

Fetuin agarose saline suspension $(500 \mu$ l, Sigma-Aldrich, Suffolk, UK) was packed in mini filter columns and equilibrated with phosphate buffer (100 mM, pH 6.8). Recombinant protein quantified by Bradford (Sigma-Aldrich) was suspended in phosphate buffer $(200 \mu \mathrm{g})$, loaded to the top of the affinity column and maintained at $4{ }^{\circ} \mathrm{C}$ for $3 \mathrm{~h}$ for complete binding [27]. The columns were washed with phosphate buffer containing $\mathrm{NaCl}$ (100 $\mathrm{mM})$ to remove non-specifically bound proteins. The fetuin-binding fraction was then eluted from the column in sodium citrate $(0.1 \mathrm{M}, \mathrm{pH}$ 6.7) and kept at $-20{ }^{\circ} \mathrm{C}$ for further analysis.

\section{MDBK cell binding assay}

MDBK cells were seeded into 24 -well plates $\left(0.3 \times 10^{6}\right.$ cells/well) and left to settle into a monolayer for $4 \mathrm{~h}$, washed gently in phosphate buffered saline (PBS), blocked in 1\% bovine serum albumin (BSA) for $30 \mathrm{~min}$, and washed again in PBS. $500 \mu \mathrm{g}$ of recombinant protein was added per well and incubated for $1 \mathrm{~h}$ at $4{ }^{\circ} \mathrm{C}$. Supernatants were removed and the cells were washed several times with PBS. MDBK monolayers were removed from wells with trypsin (Sigma-Aldrich), pelleted and stored at $-20{ }^{\circ} \mathrm{C}$ until further analysis.

\section{SDS-polyacrylamide gel electrophoresis (PAGE) and western blot}

Eluted fetuin-binding fraction $(50 \mu \mathrm{g}$ in $50 \mu \mathrm{g}$ of $2 \times$ Laemmli loading buffer (Sigma-Aldrich)) and MDBKbound protein fractions (resuspended in $100 \mu \mathrm{g} 1 \times$ Laemmli) were electrophoresed in NuPAGE 4-12\% BisTris 10-well gels (Invitrogen, California, USA) and stained with Coomassie blue (BioRad, Hertfordshire, UK). Proteins were transferred from gels to nitrocellulose membranes (GE Healthcare, Buckinghamshire, UK) by semi-dry blotting following the manufacturer's protocols (Invitrogen), and membranes were blocked in 5\% $(w / v)$ non-fat milk (Bio-Rad) overnight. Membranes were incubated for $1 \mathrm{~h}$ with mouse anti-histidine tag antibody (1/1000; Merck Millipore, Hertfordshire, UK), then washed three times with tris buffered saline-Tween20 (TBS-Tween) $0.05 \%(v / \mathrm{v})$ and incubated for $1 \mathrm{~h}$ with goat anti-mouse IgG antibody HRP conjugate (1/5000; Merck Millipore). Membranes were washed three times with TBS-Tween $0.05 \%$ (v/v), once with TBS and finally with distilled water before adding Luminata substrate (Merck Millipore). Chemiluminiscence was visualised in a G:BOX (Syngene, Cambridge, UK) and images were taken with GeneSnap 7.12 software (Syngene).

\section{Plasmid construct for the expression of EtMCP2-mCherry fusion in E. tenella}

The plasmid pMIC2-mChe [28] containing the complete coding sequence of microneme protein EtMIC2 fused to the fluorescent reporter mCherry was the starting plasmid used to generate a further plasmid containing the coding sequence of EtMCP2 fused to mCherry (pMCP2-mChe). Both plasmids contain a second expression cassette that includes the coding sequence of mCitrine for the detection and sorting by FACS of transgenic parasites. Briefly, EtMCP2 sequence lacking start and stop codons was PCR-amplified (see primers in Additional file 1: Table S1) and cloned into XbaI restriction sites in pMIC2-mChe, directly replacing EtMIC2 coding sequence but retaining EtMIC2 start codon. The PCR, sequencing, molecular 
cloning and plasmid preparation for transfection were carried out as described previously [17].

\section{Generation of transgenic populations of $E$. tenella expressing $\mathrm{mCherry}$ fusions}

Restriction enzyme-mediated integration (REMI) transfection into E. tenella sporozoites was carried out using programme EO114 of the Nucleofector 4D (Lonza ${ }^{\oplus}$, Basel, Switzerland) following protocols described previously [17]. After $24 \mathrm{~h}$ of incubation with MDBK cells, the presence of transiently transfected sporozoites expressing fluorescent reporters mCitrine and mCherry was confirmed by fluorescence microscopy (Leica Microsystems DMI3000B). To obtain stable transgenic populations (EtMIC2-mChe and EtMCP2-mChe), $0.75 \times 10^{6}$ transfected sporozoites were used to infect two chickens via the cloacal route. Shed fluorescent oocysts were selected by FACS (BD FACS Aria $^{\text {тм }}$ III) for subsequent in vivo passages to amplify the proportion of transgenic parasites within the populations.

Infected monolayers and oocysts of transgenic populations were observed under UV fluorescence in a Leica DMI3000B microscope and photographed with a Leica
DCF365FX camera. Image processing was performed using the LAS AF (Leica Microsystems).

\section{Immunofluorescence}

MDBK monolayers infected for $4 \mathrm{~h}$ with E. tenella sporozoites were fixed in $4 \%$ paraformaldehyde in PBS for $15 \mathrm{~min}$ and washed in PBS. Fixed monolayers were blocked by incubating in 3\% BSA, $0.25 \%$ Triton $\times 100$ in PBS for $30 \mathrm{~min}$ then in rabbit anti-EtMIC2 serum (1/500) for $1 \mathrm{~h}$. After three washes in PBS, monolayers were incubated with goat anti-rabbit IgG conjugated to Alexa Fluor 488 (1/1000; Invitrogen), washed in PBS, then observed under UV fluorescence using a Leica DMI3000B microscope and photographed with a Leica DCF365FX camera. Image processing was performed using the LAS AF (Leica Microsystems).

Real time quantitative PCR (qPCR) and reverse transcription qPCR (RT-qPCR)

Total DNA and RNA were extracted from an in vitro time course of parasite development in MDBK cells infected with wild-type E. tenella sporozoites following the

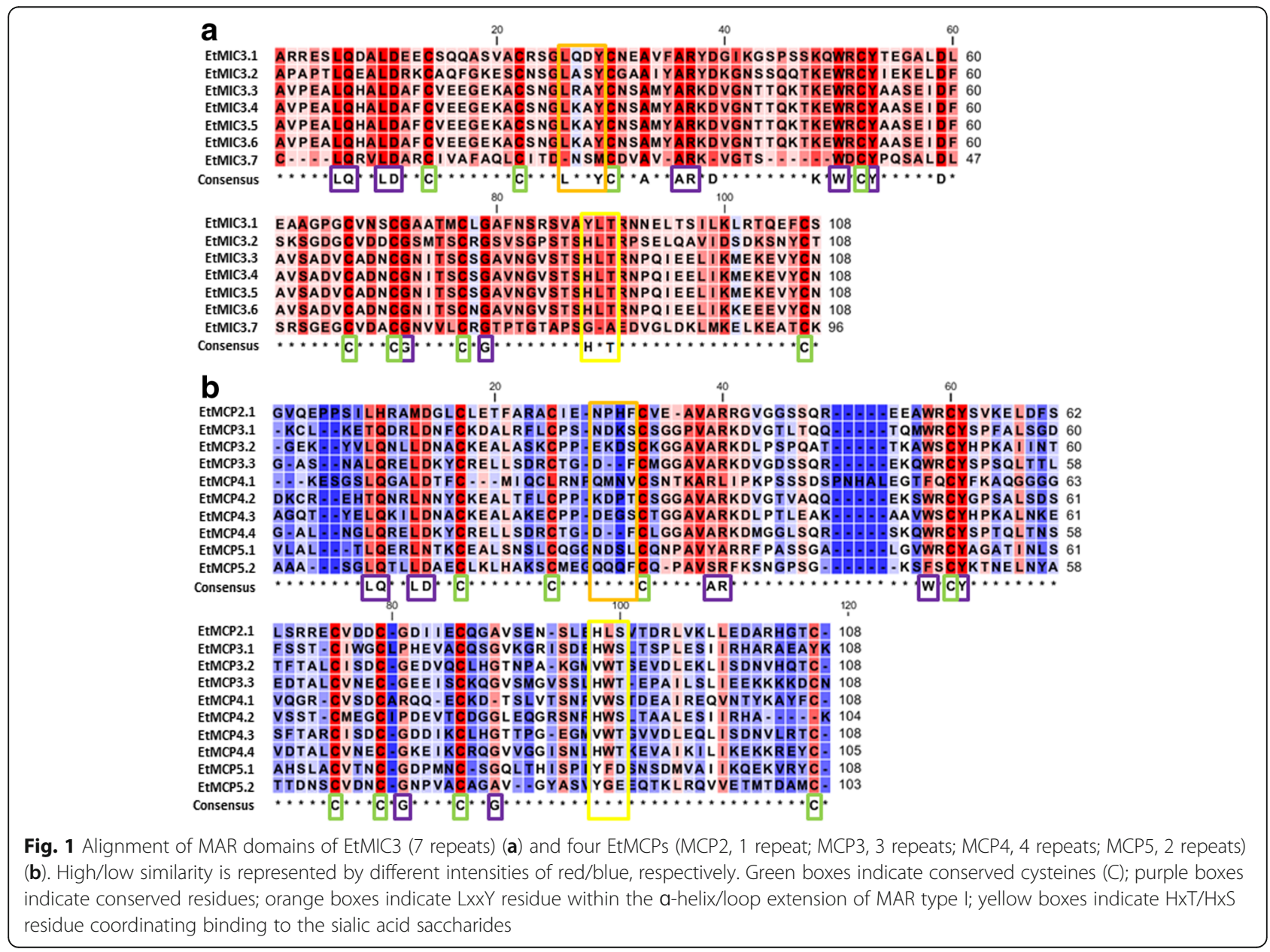


manufacturer's protocols (All prep DNA/RNA Mini Kit, Qiagen, West Sussex, UK). cDNA was synthesized from total RNA using SuperScript $\mathrm{II}^{\circ}$ reverse transcriptase (Invitrogen) as described previously [17].

Real time qPCR was performed in a CFX96 Touch $^{\circ}$ Real-Time PCR Detection System (Bio-Rad) using DNAbinding dye SsoFastTM EvaGreen ${ }^{\circ}$ Supermix (Bio-Rad) as described previously [17]. The number of haploid genomes (equivalent to sporozoites or merozoites) per well was determined for each time point using gDNA specific primers for Eimeria spp. 5S rDNA [11] and a standard curve of sporozoite gDNA extracted by the same methods. Transgene transcription was quantified from cDNA using specific primers for EtMIC2, EtMIC5, EtMCP2 and EtActin (Additional file 1: Table S1) and compared with serial dilutions of DNA standard templates for each transgene (pGEM ${ }^{\ominus}$ T Easy (Promega, Hampshire, UK) plasmid containing the EtMIC2, EtMIC5, EtMCP2 or EtActin coding sequences). Standard curves were prepared for all genes from $10^{7}$ to $10^{2}$ sporozoites or plasmid copies, against which the total number of parasites or transcript copy numbers were quantified, respectively. The transcription levels along the course of intracellular schizont development were normalised to the number of parasite genomes. Data were analysed by a one-way ANOVA with post-hoc Bonferroni test (SPSS Statistics 22, New York, USA).

\section{Results}

Type I MAR from E. tenella MCPs has low conservation in their binding domains

MAR from EtMIC3 (Fig. 1a) and from the four identified EtMCP proteins (Fig. 1b) of E. tenella were aligned in order to predict their potential binding properties (Table 1). All MAR from EtMIC3 contain eight cysteine (C) residues (Fig. 1a; green boxes) as do most MAR from EtMCPs except for EtMCP3.1 and EtMCP4.2 which lack the last one (Fig. 1b; green boxes). Most of the conserved signatures described by Blumenschein et al. [19] and Lai et al. [22] in Type I and Type II MAR are present in at least $70 \%$ of the aligned Type I MAR from EtMCPs (Fig. 1b; purple boxes). However, the LxxY signature within the a1-helix/loop extension of Type I MAR (Fig. 1a; orange boxes) that is proposed to restrict interaction with some sialyl saccharides [22] is not conserved in any MAR from the EtMCPs (Fig. 1b; orange boxes; Table 2). Moreover, the HxT motif that directly coordinates binding to sialyl saccharides (Fig.1a; yellow box) exhibits considerable variation in EtMCPs (Fig.1b; yellow box) as summarised in Table 1.

\section{Type I MAR with conserved threonine residues bind to} fetuin and to cultured cells

Variant MAR from EtMCPs (recMCP2.1/3.3/4.2/4.3/5.2) as well as MAR3 of EtMIC3 (recMIC3.3) were expressed as soluble recombinant fusion proteins in E. coli (Table 1) and tested for their ability to bind highly sialylated fetuin agarose and Madin-Darby bovine kidney (MDBK) cells. All recombinant MAR that retain the threonine residue within the HxT motif that co-ordinates sialyl binding (HLT in recMIC3.3, HWT in recMCP3.3 and VWT in recMCP4.3) bound to both fetuin agarose and MDBK cells (Fig. 2a and b, Lanes 1, 3 and 5, Table 1). All other variants that were tested (HLS in recMCP2.1, HWS in recMCP4.2 and YGE in recMCP5.2) did not bind to either target (Fig. 2a and b, Lanes 2, 4 and 6, Table 1).

\section{EtMCP2 fused to mCherry localises to the anterior end of E. tenella sporozoites in both transient and stable transgenic populations}

Sporozoites were transfected with plasmids that contain the entire coding sequences (including predicted $\mathrm{N}$ terminal signal peptides) of either EtMIC2 or EtMCP2 fused directly to $m$ Cherry (pMIC2-mChe or pMCP2mChe; Additional file 1: Figure S1) alongside a second cassette that independently expresses mCitrine. Transiently transfected sporozoites in MDBK cell cultures were found to express both reporters, with green (mCitrine) expression predominantly in the cytosol and red

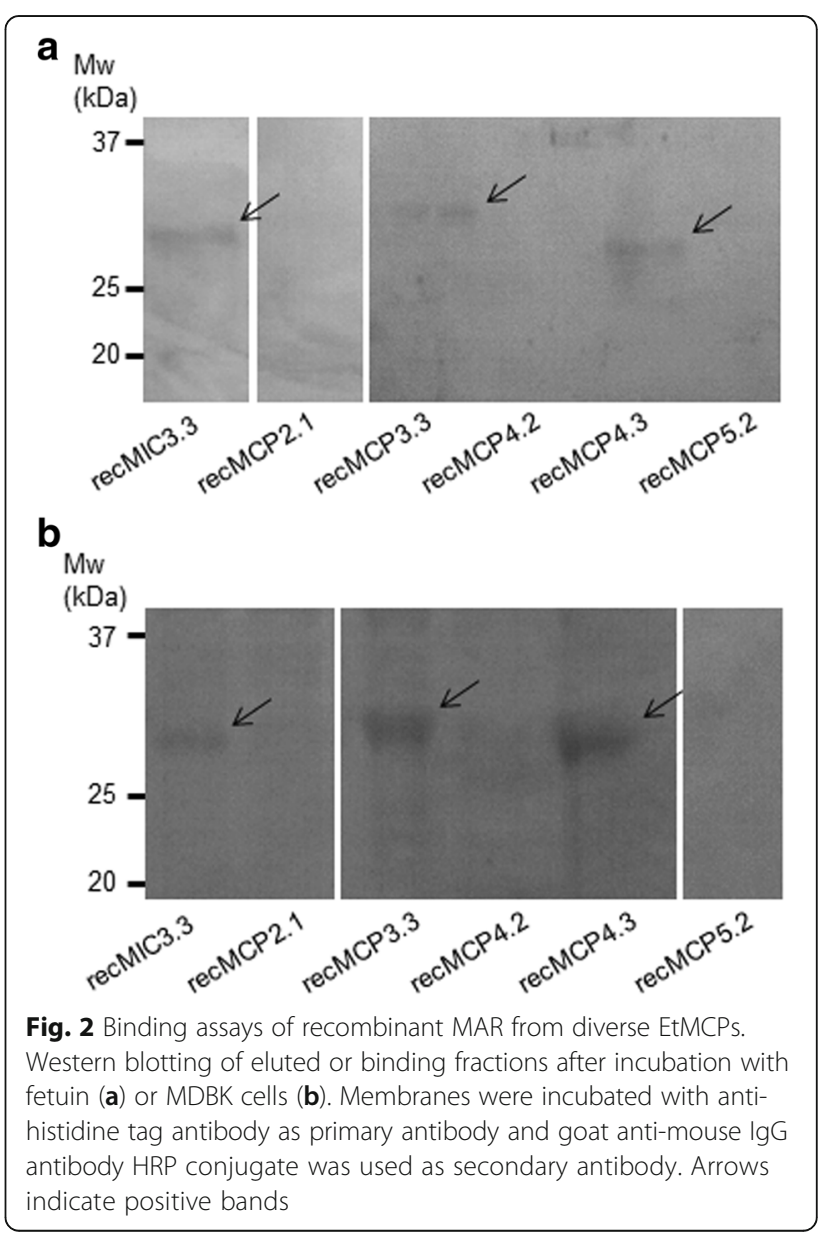


(mCherry) expression restricted to the anterior part of the sporozoite for both pMIC2-mChe and pMCP2mChe transfectants (Fig. 3a panel 1 and panel 2, respectively). EtMIC2 is a well described microneme protein [29] and its localisation when overexpressed as an mCherry fusion is similar to what is seen by immunofluorescence when MDBK monolayers are infected with wild-type sporozoites and probed with anti-EtMIC2 serum (compare Fig. 3a panel $1 \mathrm{mCherry}$ red fluorescence with panel 3 green Alexa Fluor 488 immunofluorescence). EtMCP2 fused to mCherry shows a similar pattern (Fig. 3a panel 2 mCherry red fluorescence), which allows us to conclude is also likely to be located in the micronemes.

Transfected sporozoites were used to generate stable transgenic populations (Et-MIC2-mChe and Et-MCP2mChe) by two consecutive passages through chickens with fluorescence-activated cell sorting (FACS) in between to enrich the populations in fluorescent oocysts. Within oocysts (Fig. 3b) for both populations, mCherry exhibited a different pattern of expression to that seen for cytosolic mCitrine, indicating a non-cytosolic location within the sporozoite; it was not possible to discern the precise location of expression. However, after hatching

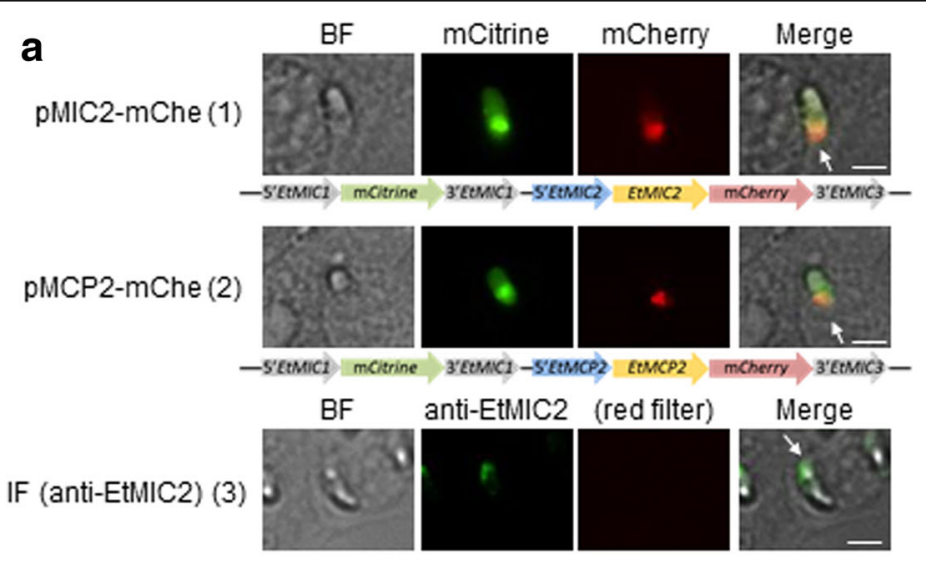

b

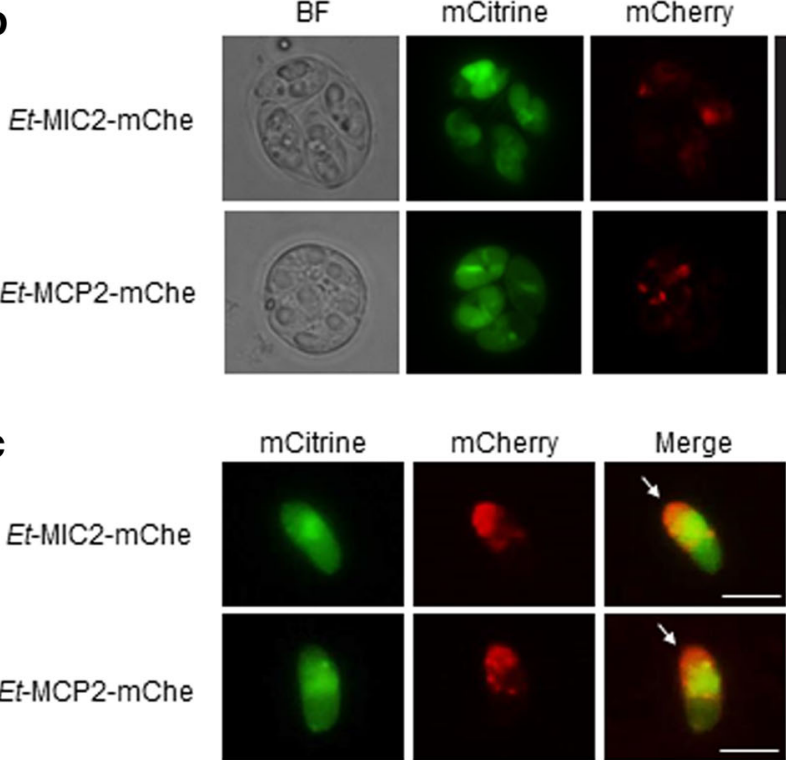

Fig. 3 Fluorescence observed in transgenic populations of E. tenella. a Transiently transfected sporozoites with MDBK cells at $24 \mathrm{~h}$ post-infection. Panel 1: pMIC2-mChe transfected sporozoites; mCherry is seen at the apical end whereas mCitrine is detected in the cytosol. Panel 2: pMCP2-mChe transfected sporozoites; mCherry is seen at the apical end part whereas mCitrine is detected in the cytosol. Panel 3: immunofluorescence (IF) of E. tenella wild type sporozoites probed with anti-EtMIC2 and goat anti-rabbit lgG conjugated to Alexa Fluor 488. The plasmid constructs for each transfection are displayed above each panel. Arrows indicate the anterior end of sporozoites. b Oocysts of stable transgenic populations Et-MIC2-mChe and Et-MCP2mChe; mCitrine was observed in the cytosol of encysted sporozoites whereas mCherry accumulated in specific patches (location cannot be assigned due the folded position of the sporozoites within the oocyst). c Sporozoites of stable transgenic populations Et-MIC2-mChe and Et-MCP2-mChe incubated with MDBK cells for $24 \mathrm{~h}$; mCherry was observed at apical end whereas mCitrine was detected the cytosol for both populations. Scale-bars: $5 \mu \mathrm{m}$ 
sporozoites of the transgenic populations and allowing these to invade MDBK cells (Fig. 3c) it could be clearly seen that fluorescence corresponded to what had been observed in transiently transfected sporozoites (Fig. 3a panels 1 and 2) with mCitrine predominantly in the cytosol and mCherry in the apical tip, as expected for microneme proteins. This pattern was similarly observed in freshly excysted sporozoites before they invaded host cells (not shown).

\section{Abundance of EtMCP2 transcripts during schizogony and comparison with genes encoding known microneme proteins}

We documented the transcript numbers per zoite of $E t M C P 2$ during endogenous parasite development in vitro by RT-qPCR and compared this to transcripts per zoite of EtMIC2, EtMIC5 (encoding two well characterised EtMIC proteins, EtMIC2 and EtMIC5 [29, 30]) and EtActin (which is expressed at a low level throughout parasite growth). In free sporozoites, EtMIC2 transcripts (Fig. 4) were at a significantly higher level than those of EtMIC5 $\left(F_{(3,8)}=22.708, P=0.012\right)$ and slightly higher, but not significantly, than those of EtMCP2 $\left(F_{(3,8)}=22.708\right.$, $P=0.066$ ). As schizogony progressed, values decreased significantly for all three genes. A very similar pattern was observed for EtMIC2 and EtMCP2, where number of transcripts per zoite were markedly reduced during schizogony (Fig. 4, 24-48 hpi), remaining at the same low level once merozoites are formed and released (48-72 hpi). In contrast, EtMIC5 transcripts per zoite were significantly higher during late endogenous development and formation and release of merozoites $\left(48-72 \mathrm{hpi} ; F_{(3,8)}=84.105\right.$,

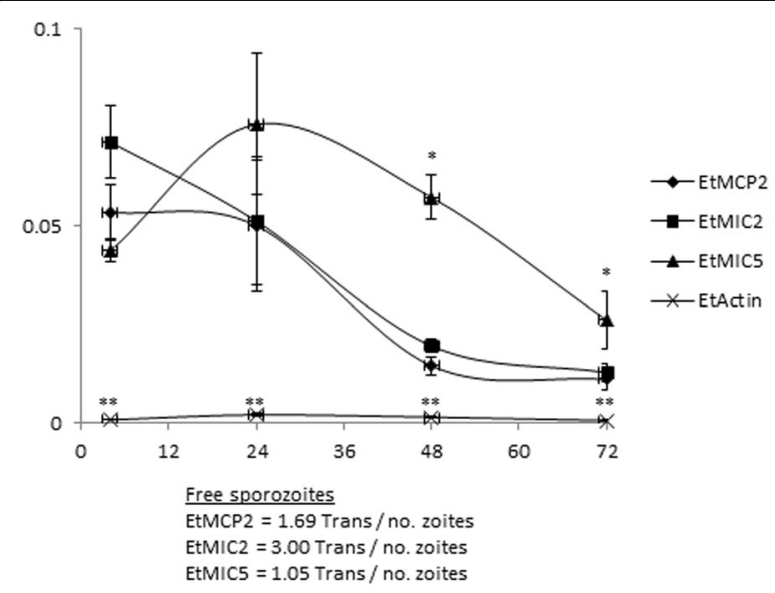

Fig. 4 Analysis of transcript abundance of E. tenella micromene proteins EtMIC2 and EtMIC5, EtActin and EtMCP2. Transcript number per zoite during a time course of intracellular development of parasites were similar for EtMIC2 and EtMCP2. Asrterisks indicate significant differences $\left({ }^{*} P<0.05,{ }^{* *} P<0.01\right.$, one-way ANOVA with Bonferroni post-hoc test). Transcription levels per zoite obtained in free purified sporozoites before invasion are shown
$P<0.0001$ and $F_{(3,8)}=28.489, P=0.019$, respectively) compared to EtMIC2 levels. As expected, EtActin was low and constant along the endogenous development.

\section{Discussion}

Microneme proteins are characterised by the possession of adhesive domains that play roles in parasite attachment and invasion of the host cell. One of these, the MAR domain, is restricted to coccidial parasites and has been shown to bind sialylated glycans. Structural and sequence variations between and within Type I and Type II MAR family domains results in variant capacities for binding sialylated residues, contributing to differences in host and site specificities of the parasites. For E. tenella the only MAR protein explored to date is microneme protein EtMIC3; this has 7 Type I MAR, is expressed by invading sporozoites, binds the surface of chicken caecal epithelial cells, and has a strong preference for terminal $\mathrm{N}$-acetylneuraminic acids with a2,3-linkages [22]. Several additional genes encoding proteins with Type I MAR domains are annotated in the E. tenella genome (EtMCP2 to EtMCP5), however, until now their adhesive properties and subcellular locations were unconfirmed.

In this study, MAR from E. tenella MCPs were analysed for their predicted and actual binding properties, along with MAR3 from EtMIC3. Blumenschein et al. [19] reported the involvement of the threonine $(\mathrm{T})$ residue in host cell carbohydrate binding; our results highlight that the threonine residue within the HxT binding domain of MAR seems to be essential for binding to fetuin or to cultured MDBK cells. None of the MAR with threonine substitutions (serine in recMCP2.1 and recMCP4.2, glutamic acid in recMCP5.2) were able to bind. However, substitution of the histidine within the HxT binding domain with a valine in recMCP4.3 was tolerated and this MAR was able to bind both targets. Interestingly, none of the EtMCP MAR domains were found contain the LxxY motif that is present in the MARI extension of the a1-helix/loop of the EtMIC3 MAR. The leucine and tyrosine residues in this motif were structurally implicated in steric hindrance within the binding pocket, resulting in EtMIC3 being able to bind only a restricted range of sialyl glycans [22]. It would be interesting therefore to explore whether the MAR from E. tenella MCP are able to bind a wider range of sialic acids, including $\mathrm{N}$-glycolyneuraminic acids, and a wider range of terminal linkages.

We aimed to determine whether EtMCP proteins are localised to the microneme organelles and chose to explore this using EtMCP2, because its small size (encompassing an $\mathrm{N}$-terminal signal peptide plus a single MAR domain) was most convenient for tagging and transfection into E. tenella. Expression of EtMCP2 fused at its Cterminus to mCherry resulted in the fused protein being 
expressed with an apical location within the sporozoite in both transiently transfected and stable transgenic populations. By comparing the pattern of expression to that seen with an analogous mCherry fusion with the characterised microneme protein EtMIC2, it seems highly likely that EtMCP2 is indeed localised to the microneme organelles where it presumably plays a role in binding to host cells.

In addition to the putative micronemal localisation, the accumulation of transcripts per zoites expressed from EtMCP2 and EtMIC2 exhibited a similar dynamic pattern during intracellular development of $E$. tenella within cultured MDBK cells. The relative numbers of transcripts continuously decreased during development until the formation of merozoites. In contrast, transcripts of another microneme encoding gene, EtMIC5, were maintained at a higher level during endogenous development.

Transgenesis in Eimeria spp. is now routinely used and most studies have focused on the use of Eimeria as a vaccine vehicle to deliver foreign antigens $[15,17,31]$. We now report the use of this technology as a convenient and rapid tool for the study of endogenous protein localization; being able to visualise the location of overexpressed proteins fused to a fluorescent reporter has allowed a previously uncharacterised MAR containing protein to be tentatively assigned to the microneme organelle. This is a promising start to further approaches to study gene functionality or use them as "bait" to capture interacting and proximal proteins, as has been recently done in Toxoplasma [32, 33].

\section{Conclusions}

The threonine (T) in the HxT motif of Type I MAR domains of E. tenella MCPs appears to play an important role in the binding properties of these proteins, as has been suggested before [21], since other variants $(\mathrm{HxS}$ and $\mathrm{YxE}$ ) did not show binding to fetuin or MDBK cells. EtMCPs are expected to be localised in the micronemes, with the aim to confirm this; EtMCP2 was expressed in transgenic Eimeria as a fusion with the fluorescent reporter mCherry exhibiting an apical localisation, typical of microneme proteins (and identical to the EtMIC2mCherry fusion). In addition, EtMCP2 showed a similar pattern of transcripts than other miconeme proteins (EtMIC2 and EtMIC5). We concluded therefore, that at least one of these newly described EtMCPs is located in the micronemes, as was expected.

\section{Additional file}

Additional file 1: Table S1. Primers used in the study. Figure S1. Plasmid construct developed and used for the genetic complementation of E. tenella. The plasmids contain a double cassette: mCitrine - green arrow - flanked by 5'EtMIC1 and 3'EtMIC1 - green arrows - and EtMIC2 and EtMCP2 fused to mCherry - yellow arrow + red arrow - flanked by $5^{\prime}$ EtMIC2 and 3'EtMIC3 - final purple arrows. (PDF $273 \mathrm{~kb}$ )

\section{Abbreviations}

BSA: bovine serum albumin; CDNA: complementary DNA; FACS: fluorescenceactivated cell sorting; MAR: microneme adhesive repeat; MCP: MAR containing proteins; MDBK: Madin-Darby bovine kidney; MIC: microneme secretory organelles; PBS: phosphate buffered saline; qPCR: quantitative PCR; REMI: restriction enzyme-mediated integration; $R T-q P C R$ : reverse transcription qPCR; TBS-Tween: tris buffered saline-Tween20

\section{Acknowledgments}

The authors would like to thank Olivia Morgan for technical assistance and advice. This manuscript has been assigned the reference PPS_01562 by the Royal Veterinary College.

\section{Funding}

This work was funded the Royal Veterinary College (JNF, OS), Biotechnology and Biological Sciences Research Council (BBSRC) Research Experience Placements and BBSRC grant reference BB/L002477/1 (VMH, FT).

\section{Availability of data and materials}

The data supporting the conclusions of this article are included within the article and its additional file.

\section{Authors' contributions}

VMH and FMT conceived and designed the experiments. VMH, OS and LP cloned expressed and performed the binding experiments of the MAR. VMH and RF generated and characterised the transgenic parasites. VMH and JNF standarised and performed the qPCR and RT-qPCR. VMH analysed the data and wrote the manuscript. FMT critically reviewed and modified the paper. All authors read and approved the final manuscript.

\section{Ethics approval and consent to participate}

This study was carried out in strict accordance with the Animals (Scientific Procedures) Act 1986, an Act of Parliament of the United Kingdom. All animal studies and protocols were approved by the Royal Veterinary College and/or MSD Animal Health Ethical Review Committees and the United Kingdom Government Home Office under specific project licence. The laboratory work involving GMO was conducted under authorization GM9708.1, administered by the UK Health and Safety Executive. The animal facilities for GMO are classified derogated containment level 3.

\section{Consent for publication}

Not applicable.

\section{Competing interests}

The authors declare that they have no competing interests.

\section{Publisher's Note}

Springer Nature remains neutral with regard to jurisdictional claims in published maps and institutional affiliations.

Received: 9 May 2017 Accepted: 5 October 2017

Published online: 17 October 2017

\section{References}

1. Blake DP, Tomley FM. Securing poultry production from the ever-present Eimeria challenge. Trends Parasitol. 2014;30:12-9.

2. McDonald V, Shirley MW. Past and future: vaccination against Eimeria. Parasitology. 2009;136:1477-89.

3. Lal K, Bromley E, Oakes R, Prieto JH, Sanderson SJ, Kurian D, et al. Proteomic comparison of four Eimeria tenella life-cycle stages: unsporulated oocyst, sporulated oocyst, sporozoite and second-generation merozoite. Proteomics. 2009;9:4566-76.

4. Oakes RD, Kurian D, Bromley E, Ward C, Lal K, Blake DP, et al. The rhoptry proteome of Eimeria tenella sporozoites. Int J Parasitol. 2013:43:181-8.

5. Reid AJ, Blake DP, Ansari HR, Billington K, Browne HP, Bryant J, et al. Genomic analysis of the causative agents of coccidiosis in domestic chickens. Genome Res. 2014;24:1676-85. 
6. Jimenez-Ruiz E, Wong EH, Pall GS, Meissner M. Advantages and disadvantages of conditional systems for characterization of essential genes in Toxoplasma gondii. Parasitology. 2014;141:1390-8.

7. Besteiro S, Dubremetz JF, Lebrun M. The moving junction of apicomplexan parasites: a key structure for invasion. Cell Microbiol. 2011;13:797-805.

8. Tomavo S, Slomianny C, Meissner M, Carruthers VB. Protein trafficking through the endosomal system prepares intracellular parasites for a home invasion. PLoS Pathog. 2013;9:e1003629.

9. Bargieri DY, Andenmatten N, Lagal V, Thiberge S, Whitelaw JA, Tardieux I, et al. Apical membrane antigen 1 mediates apicomplexan parasite attachment but is dispensable for host cell invasion. Nat Commun. 2013;4:2552.

10. Lei T, Wang H, Liu J, Nan H, Liu Q. ROP18 is a key factor responsible for virulence difference between Toxoplasma gondii and Neospora caninum. PLoS One. 2014;9:e99744

11. Clark JD, Billington K, Bumstead JM, Oakes RD. Soon PE, Sopp P, et al. a toolbox facilitating stable transfection of Eimeria species. Mol Biochem Parasitol. 2008:162:77-86.

12. Yan W, Liu X, Shi T, Hao L, Tomley FM, Suo X. Stable transfection of Eimeria tenella: constitutive expression of the YFP-YFP molecule throughout the life cycle. Int J Parasitol. 2009;39:109-17.

13. Huang X, Zou J, Xu H, Ding Y, Yin G, Liu X, Suo X. Transgenic Eimeria tenella expressing enhanced yellow fluorescent protein targeted to different cellular compartments stimulated dichotomic immune responses in chickens. J Immunol. 2011;187:3595-602.

14. Liu X, Zou J, Yin G, Su H, Huang X, Li J, et al. Development of transgenic lines of Eimeria tenella expressing M2e-enhanced yellow fluorescent protein (M2e-EYFP). Vet Parasitol. 2013;193:1-7.

15. Clark JD, Oakes RD, Redhead K, Crouch CF, Francis MJ, Tomley FM, Blake DP. Eimeria species parasites as novel vaccine delivery vectors: antiCampylobacter jejuni protective immunity induced by Eimeria tenelladelivered CjaA. Vaccine. 2012;30:2683-8.

16. Yin G, Qin M, Liu X, Suo J, Suo X. Expression of Toxoplasma gondii dense granule protein7 (GRA7) in Eimeria tenella. Parasitol Res. 2013;112:2105-9.

17. Marugan-Hernandez V, Cockle C, Macdonald S, Pegg E, Crouch C, Blake DP, Tomley FM. Viral proteins expressed in the protozoan parasite Eimeria tenella are detected by the chicken immune system. Parasit Vectors. 2016;9:463.

18. Carruthers VB, Tomley FM. Microneme proteins in apicomplexans. Subcell Biochem. 2008:47:33-45.

19. Blumenschein TM, Friedrich N, Childs RA, Saouros S, Carpenter EP, Campanero-Rhodes MA, et al. Atomic resolution insight into host cell recognition by Toxoplasma gondii. EMBO J. 2007;26:2808-20.

20. Friedrich N, Santos JM, Liu Y, Palma AS, Leon E, Saouros S, et al. Members of a novel protein family containing microneme adhesive repeat domains act as sialic acid-binding lectins during host cell invasion by apicomplexan parasites. J Biol Chem. 2010;285:2064-76.

21. Cowper B, Matthews S, Tomley F. The molecular basis for the distinct host and tissue tropisms of coccidian parasites. Mol Biochem Parasitol. 2012;186:1-10.

22. Lai L, Bumstead J, Liu Y, Garnett J, Campanero-Rhodes MA, Blake DP, et al. The role of sialyl glycan recognition in host tissue tropism of the avian parasite Eimeria tenella. PLoS Pathog. 2011;7:e1002296.

23. Lai L, Simpson P, Bumstead J, Tomley F, Matthews S, Complete NMR. Assignments for the second microneme adhesive repeat (MAR) domain from Eimeria tenella microneme protein EtMIC3. Biomol NMR Assign. 2009;3:175-7.

24. McDougald $L R$, Jeffers TK. Comparative in vitro development of precocious and normal strains of Eimeria tenella (Coccidia). J Protozool. 1976;23:530-4.

25. Long PL, Millard BJ, Joyner LP, Norton CCA. Guide to laboratory techniques used in the study and diagnosis of avian coccidiosis. Folia Vet Lat. 1976;6:201-17.

26. Shirley M. Eimeria species and strains of chickens. In: Eckert J, Braun R, Shirley M, Coudert P, editors. Guidelines on techniques in coccidiosis research. Luxembourg: European Commission; 1995. p. 1-51.

27. Vonlaufen N, Naguleswaran A, Gianinazzi C, Hemphill A. Characterization of the fetuin-binding fraction of Neospora caninum tachyzoites and its potential involvement in host-parasite interactions. Parasitology. 2007;134:805-17.

28. Marugan-Hernandez V, Long E, Blake D, Crouch C, Tomley F. Eimeria tenella protein trafficking: differential regulation of secretion versus surface tethering during the life cycle. Sci Rep. 2017;7:4557.

29. Tomley FM, Bumstead JM, Billington KJ, Dunn PP. Molecular cloning and characterization of a novel acidic microneme protein (Etmic-2) from the apicomplexan protozoan parasite, Eimeria tenella. Mol Biochem Parasitol. 1996;79:195-206.
30. Brown PJ, Billington KJ, Bumstead JM, Clark JD, Tomley FMA. Microneme protein from Eimeria tenella with homology to the apple domains of coagulation factor XI and plasma pre-kallikrein. Mol Biochem Parasitol. 2000;107:91-102.

31. Tang X, Yin G, Qin M, Tao G, Suo J, Liu X, Suo X. Transgenic Eimeria tenella as a vaccine vehicle: expressing TgSAG1 elicits protective immunity against Toxoplasma gondii infections in chickens and mice. Sci Rep. 2016;6:29379.

32. Chen AL, Kim EW, Toh JY, Vashisht AA, Rashoff AQ, Van C, et al. Novel components of the Toxoplasma inner membrane complex revealed by BiolD. MBio. 2015;6:e02357-14.

33. Nadipuram SM, Kim EW, Vashisht AA, Lin AH, Bell HN, Coppens I, et al. vivo iotinylation of the Toxoplasma parasitophorous vacuole reveals novel dense granule proteins important for parasite growth and pathogenesis. MBio. 2016; $7:$ :00808-16

\section{Submit your next manuscript to BioMed Central and we will help you at every step:}

- We accept pre-submission inquiries

- Our selector tool helps you to find the most relevant journal

- We provide round the clock customer support

- Convenient online submission

- Thorough peer review

- Inclusion in PubMed and all major indexing services

- Maximum visibility for your research

Submit your manuscript at www.biomedcentral.com/submit
Biomed Central 